\title{
Parabolic pulse evolution in normally dispersive fiber amplifiers preceding the similariton formation regime
}

\author{
Christophe Finot, Francesca Parmigiani, Periklis Petropoulos, David J. Richardson \\ Optoelectronics Research Centre, University of Southampton, Southampton SO 17 1BJ, United-Kingdom
}

We show analytically and numerically that parabolic pulses and similaritons are not always synonyms and that a self-phase modulation amplification regime precedes the self-similar evolution. Properties of the recompressed pulses after SPM amplification are investigated.

\section{Introduction}

Self-similar propagation in high-power fiber amplifiers has generated considerable interest since the first theoretical and experimental demonstration of the phenomena in 2000 [1]. Indeed, due to the combination of normal dispersion, non-linearity and gain, any initial waveform will evolve asymptotically into a similariton, a pulse characterized by a parabolic intensity profile with a linear positive chirp. This pulse then undergoes self-similar (SS) dynamics with a longitudinal exponential increase in the peak power and the temporal and spectral widths. The only issue is simply one of the time/ length scales required to achieve this.

To date, no clear difference has been made in the literature between self-similar evolution and the amplification of pulses that simply have a parabolic input shape. However, with the advances in similariton lasers [2], and the use of linear pulse shaping, using for example superstructured fiber Bragg gratings [3], it has become possible to use a parabolic pulse as the input to an amplifier. The generalised expression for parabolic pulse is given by:

$$
\psi_{p}(t)=A_{P} \sqrt{1-2 \frac{t^{2}}{T_{P}^{2}}} \exp \left(-i \frac{C_{P}}{2} \cdot t^{2}\right) \quad \text { if }|t| \leq T_{P} / \sqrt{2}, \quad \psi_{P}(t)=0 \text { otherwise, }
$$

where $A_{P}$ is the amplitude of the pulse, $T_{P}$ the FWHM temporal width and $\mathrm{C}_{P}$ the linear chirp coefficient.

We demonstrate herein that the dynamics involved in the amplification of parabolic pulses can be distinctly different from the self-similar evolution usually referred to when speaking about parabolic amplification. We show that the dynamics in the initial stages of pulse evolution is not self-similar at all, but dominated by self-phase modulation (SPM). Only after a sufficient propagation length do the pulses become self similar. We also investigate the pulse properties after linear compression and show that parabolic pulses represent the optimal initial pulse shape in terms of compressed pulse quality for the SPM amplification regime.

\section{Self-phase modulation and self-similar amplification regimes}

We consider the amplification of a pulse with an initial full-width at half-maximum temporal width $T_{O}$, an initial energy $U_{0}$, either with or without an initial temporal linear chirp with a positive slope coefficient $C_{O}$. The evolution of the complex electric field $\psi$ can then be modelled by the non-linear Schrödinger equation including a constant gain term $g$, the second order dispersion $\beta_{2}$ and the non-linear coefficient $\gamma$ :

$$
i \frac{\partial \psi}{\partial z}=i \frac{g}{2} \psi+\frac{\beta_{2}}{2} \frac{\partial^{2} \psi}{\partial t^{2}}-\gamma|\psi|^{2} \psi
$$

We first consider the amplification of $800 \mathrm{fs}$ pulses. We can see in Fig. 1a that these pulses quickly converge to the SS solution (circles) which corresponds to a parabolic pulse with parameters $T_{S S}$ and $C_{S S}$ given by :

$$
T_{S S}(z)=3 \sqrt{2} U_{O}^{1 / 3} \frac{\sqrt[3]{\beta_{2} \frac{\gamma / 2}{2}}}{g^{2 / 3}} \exp \left(\frac{g}{3} z\right) \quad \text { and } \quad C_{S S}=\frac{g}{3 \beta_{2}} .
$$

regardless of the initial shape [1]. For longer initial pulses, the distance needed to converge to the asymptotic SS behaviour becomes longer. Indeed, there is a vast region where longer pulses do not exhibit any change in their temporal width. By increasing the temporal width by 10 , the ratio between the initial non-linear and dispersive lengths decreases by a factor of 10 , such that in this region SPM becomes the dominant effect. The evolution of the initial parabolic pulse characteristics can then be evaluated by :

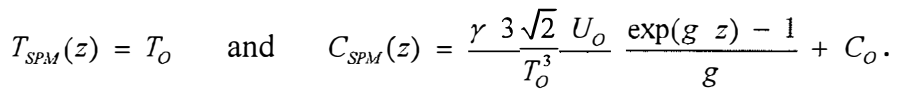

The difference between the SPM and SS amplification regime can also clearly be seen in Fig $1 \mathrm{~b}$ and $1 \mathrm{c}$ in which the longitudinal evolutions of chirp coefficient and spectral width of the pulse during the amplification are 
presented. The figure highlights the facts that the amplification of longer parabolic pulses is dominated by SPM at the beginning of the propagation (diamonds, Eq. 4) and then by SS evolution (circles, Eq. 3).
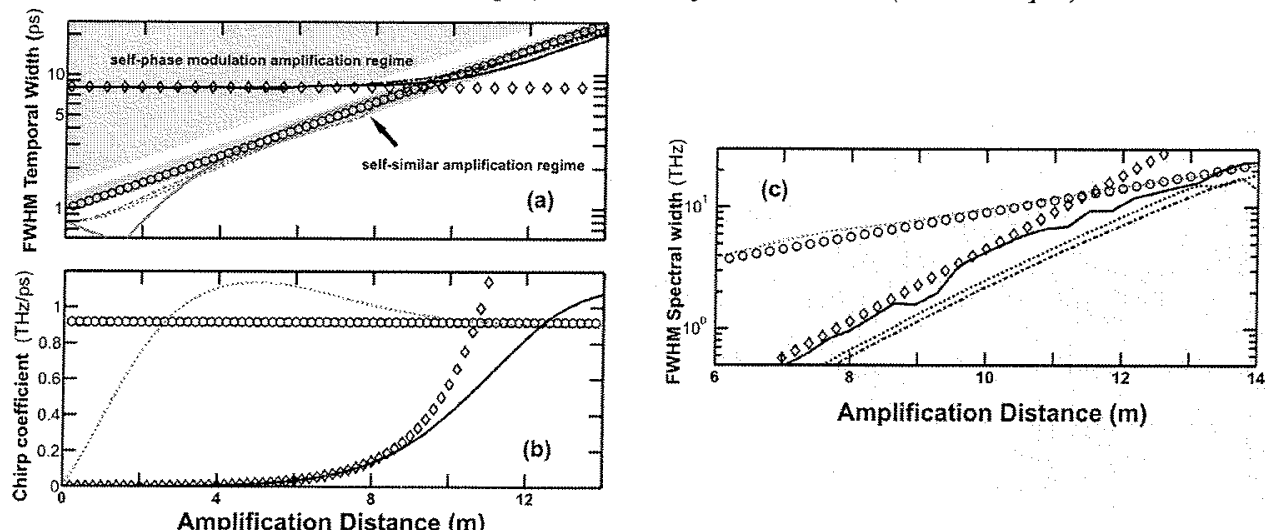

Amplification Distance (m)

Amplification Distance (m)

Fig. 1. Longitudinal evolution of different initial chirp-free pulses with an initial energy $U_{0}=50 \mathrm{pJ}$. Different initial shapes (Gaussian, sech and parabolic, solid, dotted and mixed lines respectively) and different initial FWIM temporal width ( 800 fs and 8 ps, grey and black lines respectively) are considered Analytical results in the SS regime (circles, Eq. 3) and in the SPM regime (diamonds, 8 ps parabolic initial pulse, $E q$. 4) are compared with the numerical integration of Eq. (2). The parameters of the amplifier at $1550 \mathrm{~nm}$ are $\beta_{2}=40.10-3 \mathrm{ps}^{2} . \mathrm{m}^{-1}$, $\gamma=6.10^{-3} \mathrm{~W}^{-1} \cdot \mathrm{m}^{-1}$ and $\mathrm{g}=3 \mathrm{~dB} \cdot \mathrm{m}^{-1}$. (a) Evolution of the temporal width. (b) Longitudinal evolution of the linear chirp parameter $C_{P}$ (c) Longitudinal evolution of the spectral width. The FWHM spectral width of a parabolic is given by: $f_{p}=C_{p} T_{P} / 2 \pi$

\section{Recompression}

We also study the quality of compression that can be achieved in the SPM regime. The initial parabolic pulse shape leads to the best quality pulses, exhibiting only small pedestals. Indeed, the intensity of a perfect parabolic pulse after recompression [4] is proportional to the $J_{I}(x) / x$ function, a function which is also involved in the well-known Fraunhoffer diffraction pattern of a circular aperture. A detailed expression for the parameters of the recompressed pulse obtained by recompression of the SPM-amplified pulse is given in Fig 2. As can be seen, using an initial parabolic shape, less energy remains in the wings (Fig. 2a) and the temporal width of the recompressed pulse is shorter (Fig. 2b) compared to the results obtained with either Gaussian or sech pulses. As a result, the peak-power of the pulses after recompression is significantly enhanced by using an initial parabolic shape (Fig. $2 \mathrm{c}$ ).
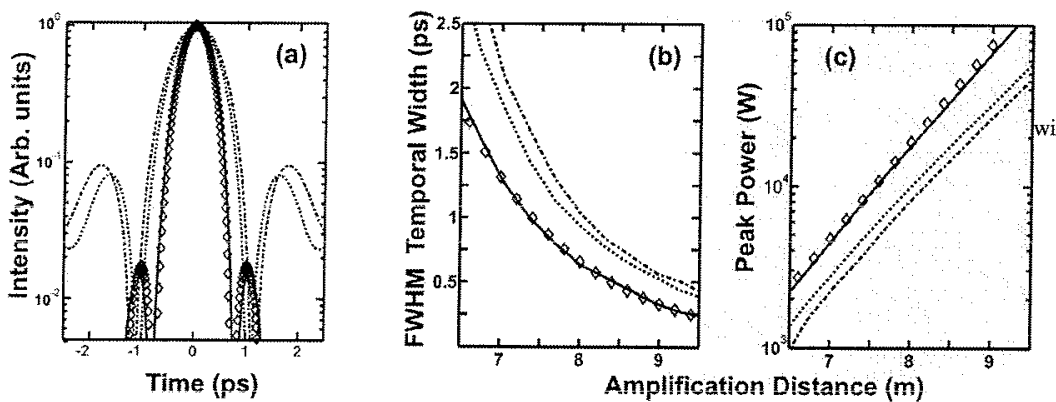

$$
\psi_{C}(t) \propto \frac{J_{1}\left(\sqrt{2} \pi f_{P} t\right)}{\sqrt{2} \pi f_{P} t}
$$

with $J_{1}$ first order Bessel function of the first kind

$$
\begin{gathered}
T_{C_{-} S P M}=\frac{1}{1.33 f_{S P M}} \\
P_{C_{-} S P M M}=\frac{3 \pi}{4 \sqrt{2}} U_{o} \Gamma^{2}\left(\frac{3}{2}\right) f_{S P M} \exp (g z) \\
\text { with } \Gamma \text { the gamma function. }
\end{gathered}
$$

Fig. 2. Properties of the recompressed pulse after amplification for different 8 ps input pulse shapes (same conventions as in Fig 1 ). The analytical predictions obtained in the SPM regime are compared to the numerical simulations. (a) Intensity profiles of the recompressed pulses after 8 meters of amplification (peak intensity normalized to l) (b) Evolution of the FWHM temporal width of the recompressed pulses. (c) Evolution of the peak-power $P_{C}$ of the recompressed pulse.

\section{Conclusion}

We have studied the amplification of parabolic pulses outlining that for larger pulse widths an SPM dominated regime precedes the SS regime. Analytical and numerical results show that the evolution and the properties of the parabolic pulses in this regime are distinctly different from those of similaritons. Compared to Gaussian or sech pulses, parabolic pulses amplified under the SPM regime can lead to a significant improvement in the recompression process. These results may have major implications in the development of parabolic amplifier chains.

\section{References}

1. M.E. Fermann, et al., Self-similar propagation and amplification of parabolic pulses in optical fibers, Phys. Rev. Lett. 84, 6010-6013, (2000).

2. F.Ö. Ilday, et al., Self-similar evolution of parabolic pulses in a laser, Phys. Rev. Lett. 92, 213902, (2004).

3. F. Parmigiani, et al., A novel XPM based pulse retiming system incorporating a fiber grating based parabolic pulse shaper, in ECOC 2005 .

4. A.C. Peacock, Self-similar amplification and propagation of parabolic pulses in optical fibers, 2001, Master thesis at Auckland University, NZ. 